\title{
Etude comparative des effets de l'alimentation des enfants à base de trois rations différentes (le maïs QPM, le maïs ordinaire et le maïs ordinaire mélangé au soja), dans la Cité de Mwene Ditu en RDC
}

Kamukenji Nam'a Mbaji Alphonse1, Kabanga Mukendi Jean Jacques², Kaseba Kabamba Stephane ${ }^{1}$, Mukendi Kamambo Robert ${ }^{5}$, Kamuevu Kamukenji Edouard ${ }^{3}$, Tshimanga Emanuel ${ }^{4}$, Tshiamala Ngeleka Theophile ${ }^{1}$.

1 : Institut National Pour l'étude et la recherche agronomiques (INERA), centre de recherche de Ngandajika (RDC)

2 : Institut Supérieur de Techniques Médicales (ISTM) Ngandajika (RDC)

3 : Institut Supérieur de Sciences Médicales (ISSM) de Mwene Ditu.

4 : Université Officielle de Mbuji-Mayi (UOM)

5 : Institut Supérieur d'étude Agronomique de Mukongo (ISEA);

Email correspondant : alphonsekamukenj@gmail.com

Original submitted in on 20 $0^{\text {th }}$ May 2019. Published online at www.m.elewa.org/journals/ on $31^{\text {st }}$ December 2019 https://doi.org/10.35759/JABs.v144.1

\section{RESUME}

Objectif du travail: La récupération des enfants mal nourris et kwashiorkorés ou marasmiques exige l'utilisation des aliments en quantité et en qualité bien équilibrées. L'objectif de ce travail consistait à trouver un aliment disponible, à la portée de tous et bon marché et à base des produits locaux.

Méthode et résultats : Le dispositif expérimental a consisté à répartir les 48 enfants sélectionnés en quatre groupes différents selon les quatre traitements suivants, par quartier et pendant 3 semaines. Ces aliments étaient distribués sous forme de bouillie chaque matin entre $7 \mathrm{~h} 30$ et $8 \mathrm{~h} 00$.

Traitement 1 : Le Quality Protein Maize (QPM) : maïs à haute valeur protéique)

Traitement 2 : Le maïs ordinaire associé au Soja,

Traitement 3 : Le maïs ordinaire,

Traitement 4 : le témoin, représenté par les enfants non soumis au traitement.

Conclusion et application des résultats : l'analyse des moyennes en terme de gain de poids observés chez les enfants dans chaque traitement est répété trois fois en fonction des nombre des quartiers considérés. L'objectif est de voir l'influence de chaque traitement sur l'évolution des poids chez les enfants mal nourris soumis aux différents traitements et déterminer le meilleur aliment parmi ces quatre. L'analyse des moyennes en termes de gain de poids observés chez les enfants après traitement, montre que la ration constituée du traitement des enfants au maïs QPM a donné des bons résultats et a montré une différence hautement significative sur les autres traitements. Ce maïs (QPM) peut donc être utilisé pour la récupération des enfants en très mauvaise santé.

Mots clés : QPM : (maïs à haute valeur protéique) 


\section{ABSTRACT}

Objective: To recuperate children suffering of malnutrition (kwashiorkor or stagnation) requires the use of food in quality and quantity well-balanced. The objective of this work was to find an available food, at the reach of all and cheap and from local products.

Method and results: The experimental device has consisted to divide 48 selected children into different groups according four following treatments, by area during three weeks. This food was distributed in form of baby's cereal every morning between $7 \mathrm{~h} 30^{\prime}$ and $8 \mathrm{~h} 00$.

Treatment 1: Quality protein maize (QPM).

Treatment 2: Ordinary maize mixed with soya bean.

Treatment 3: Ordinary maize only Common mays,

Treatment 4: Children not subjected to the treatment represent the Control.

Conclusion and results application: The analysis of averages in weight gain observed in every treatment is repeated three times according to the number of considered areas. The objective is to remark the influence of every treatment on weight evolution of children suffering of treatments and to determine the best food among these four. The averages analysis of weight gain observed for malnourished children after treatment shows that the food intake constituted of children fed with QPM has given good results and has shown a highly significant difference on other treatments. This maize (QPM) may be used to recuperate children with bad health.

Keywords: QPM: Quality Protein Maize

\section{INTRODUCTION}

On estime actuellement dans le monde 500.000 .000 le nombre des personnes insuffisamment nourries et à 1.500.000.000 le nombre des personnes qui soufrent de conséquences d'une alimentation insuffisamment équilibrée (malnutrition ; faim des protéines) (FAO, 2016). Le problème de sous-alimentation ou de malnutrition concerne en premier lieu le groupe le plus vulnérable: les enfants et les personnes âgées. Près de 800.000 .000 de personnes dans le monde souffrent de la fin chronique et 2000000 sont mal nourris et souffrent de carence en micro nutriments (FAO 2017).La malnutrition ne se manifeste pas seulement par le retard de croissance ou la maigreur, mais peut aussi se traduire par un surpoids ou par l'obésité qui constituent des problèmes émergents et sérieux pour certains enfants (Meijers J.M, et al., 2010). II semble bien que les conséquences de la malnutrition soient d'autant plus irréversibles que la malnutrition est plus précoce. Le nombre de personnes sous-alimentées dans le monde se rapproche du milliard et la situation pourrait empirer, selon la $\mathrm{FAO}$, en raison de la crise économique et financière actuelle. En effet, plus de 40 millions de personnes supplémentaires ont rejoint en 2008 « les rangs des sous-alimentés de la planète du fait principalement de la hausse des prix des denrées alimentaires ». (Rapport de I'OMS, Bureau Régional pour l'Afrique, Novembre 2016) 2016 des personnes sous alimentées vivent dans les pays en développement. Sept pays rassemblent $65 \%$ des affamés: Inde, Chine, République démocratique du Congo, Bangladesh, Indonésie, Pakistan et Ethiopie. 25000 personnes, surtout des enfants, meurent chaque jour de la malnutrition protéino-énergétique (MPE). Sur 62 millions de personnes qui meurent en moyenne chaque année, 36 millions, soit $58 \%$ directement et ou indirectement de suite de carences nutritionnelles, d'infections ou de maladies dans un contexte de sous-alimentation. Le nombre de personnes sous-alimentées a augmenté de 34 millions en Afrique subsaharienne et de 15 millions en Asie sud-est. La proportion des enfants sousalimentés de moins de 5 ans en Asie est passé de 6 à $9 \%$, alors que ce taux qui est de $32 \%$ en Afrique a peu évolué (Pierre Aubry, 2009). Le retard de croissance ou insuffisance de croissance et de développement se produit quand les enfants souffrent de malnutrition, de maladie et du manque 
de stimulation psychosocial. II survient en général avant que l'enfant n'atteigne l'âge de deux ans, et les conséquences à long terme comprennent le mauvais rendement scolaire, les bas salaires à l'âge adulte, la perte de productivité et le risque accru de maladies chroniques liées à la nutrition chez les adultes. (Rapport de l'OMS, Bureau Régional pour l'Afrique, Novembre 2016). La malnutrition est une cause sous-jacente de décès par maladies infectieuses chez l'enfant dans les pays en développement, en général, et en République Démocratique du Congo en particulier. Elle est la résultante d'une alimentation inadéquate due à des pratiques alimentaires inappropriées et aux maladies infectieuses et parasitaires qui se développent dans des conditions d'hygiène environnementale, individuelle et collective déficientes. En République Démocratique du Congo, après trois décennies de crise socioéconomique et politique, crise exacerbée par des conflits armés à répétition, la situation nutritionnelle de la population s'est gravement détériorée. Dans la ville de Mwene Ditu et ses environs, le même problème de la sous-alimentation et de la malnutrition de l'enfant se pose avec autant plus de rigueur qu'il faut trouver dans le plus bref délai le moyen d'arrêter rapidement ces maladies, morbidités et les mortalités infantiles causées par la sous-alimentation et la malnutrition chronique. Depuis un certain temps, Utilisation de soja dans la lutte contre la malnutrition infantile a réussi pendant une certaine période relativement longue, mais le prix du soja sur le marché n'a fait qu'augmenter en suivant la courbe de celui de la viande. Ce qui a rendu l'utilisation du soja encore plus rare. Cependant, la plupart des aliments protidiques utilisées dans la nutrition des enfants et personnes mal nutris, qu'ils soient d'origine animale (viande, poissons, lait ainsi que d'autres produits carnés et laitiers) ou végétale (soja surtout et un peu le haricot) sont souvent très chers et non

\section{MATERIEL ET METHODE}

Milieu d'étude: Zone à vocation agricole par excellence, après Ngandajika, pour la Province de la LOMAMI, la ville de Mwene Ditu se situe à $60^{\circ} 45^{\prime}$ (Ouest) de latitude, $23^{\circ} 57^{\prime}$ (Est) de longitude et à 700 disponibles pour le petit peuple. A titre exemplatif, $1 \mathrm{Kg}$ de viande coûte entre 8 et $9 \$$ sur le marché de Ngandajika, Mwene Ditu et même à Mbuji Mayi, compères Kamukenji, 2018. C'est pour cette raison qu'il s'avère indispensable de trouver un produit qui soit disponible à tout moment, moins cher et à la portée de toute la population rurale. Cette alimentation reste la clé quant à la construction de l'organisme des enfants grâce aux vitamines et aux sels minéraux .Elle doit contenir les éléments nécessaires jouant ainsi le rôle de la sentinelle. Elle doit apporter également l'énergie nécessaire pour le fonctionnement convenable des muscles (Rotsar et Courtejoie, 1975). Le maïs est le produit le plus disponible et le moins cher dans nos milieux et son utilisation peut satisfaire largement les besoins des enfants, mais malheureusement, le maïs et très déficitaire en acides aminés essentiels et ses protéines présentent une faible valeur biologique comparativement à l'œuf dont la valeur biologique est considérée comme valeur de référence et fixée à $100 \%$. Pourtant, le maïs QPM est un maïs qui possède les acides aminés essentiels (lysine et tryptophane) faisant défaut dans d'autres maïs et dont la valeur biologique s'approche à celle de l'œuf (90\% celle de l'œuf) (Kankolongo Mbuya, 2015). C'est dans ce cadre que nous nous sommes proposé de mener sur la cité de Mwene Ditu, une étude comparative des effets du maïs QPM, du maïs ordinaire seul, du maïs ordinaire mélangé au soja, sur le développement du poids, du périmètre brachiale et de la taille des enfants. La seule source disponible et la plus importante dans l'alimentation humaine est constituée par le maïs qui constitue la source principale d'énergie et de protéines $(7-13 \%$ de protéines mais de valeur biologique faible $40 \%$ celle du lait). II s'avère donc nécessaire de trouver une autre source alimentaire contenant des protéines d'une haute valeur biologique et pouvant fournir suffisamment d'énergie.

m d'altitude. La pluviosité moyenne arrive à $1500 \mathrm{ml}$ de pluie/an et les températures moyennes de 29 degré pour la température maximale et de 19 degré pour les températures minimales. Deuxième ville de la province 
de Lomami, après Kabinda (Chef-lieu de la province), elle est située à environ $260 \mathrm{~km}$ de la ville de Kabinda et à environ $110 \mathrm{~km}$ de la cité de Ngandajika.

Climat : Le climat général de la contrée est du type $A_{w 3}$ selon la classification climatique de Koppen, caractérisé par deux saisons. La grande saison de pluies va théoriquement du 15 Août au mois de Décembre et la petite saison de pluies va du 15 Janvier au 15 Mai. La grande saison sèche va du 15 Mai au 15 Août, alors que la petite commence au mois de Décembre jusqu'au 15 Janvier.

Végétation: Elle est essentiellement constituée des savanes guinéennes herbeuses, à dominance d'Imperata cylindrica (Crabbe et Totiwe, 1979), boisées et entrecoupées par endroit des galeries forestières (ou massifs isolés) semi-décidues, subéquatoriales et guinéennes le long des cours d'eaux (rivières et ruisseaux). La majeure partie du territoire est constituée de groupements où dominent les espèces telles: Imperata cylindrica, Hyparrhenia dissoluta, Digitaria brazzol, Triunfetta musterau et Eriosema griseum (Anonyme, 2003)

Sols: La zone agricole dont fait partie la ville de Mwene Ditu se caractérise par un faciès de savanes à Hyparrhenia sur sols bons à moyens (majoritairement des ferralsols sur sable de Kalahari. (FAO/UNESCO, 1977 ; FAO, 1999). Le sol du site de Mwene Ditu est sablo-argileux et présente une teneur élevée en sable et très peu d'éléments fins.

\section{Matériel}

Matériel humain : L'expérience a été effectuée sur les enfants dont l'âge varie entre deux à cinq ans, choisis dans les familles qui ont accepté de nous céder leurs enfants pour cet exercice. Tous ces enfants appartiennent à des différents quarties de la ville de
Mweneditu, à savoir Makota, Tshibangu Mpata et Kamisangi. 48 enfants ont été sélectionnés dans cette étude, à raison de 12 enfants répartis dans 4 Communes différentes.

Rations utilisées :

- Bouillie de la farine de QPM,

- Bouillie de la farine de maïs ordinaire associé au Soja

- Bouillie de maïs ordinaire seul.

Autres matériels utilisés : En plus de 48 enfants, les matériels ci-après ont été utilisés pour les mensurations :

Un ruban métrique et une toise à deux branches pour la mesure de la taille des enfants et le périmètre brachiale.

Le peson (balance) pour la prise de poids.

METHODE.

Dispositif expérimental : Le dispositif expérimental a consisté à répartir les 48 enfants sélectionnés en quatre groupes différents selon les quatre traitements suivants, par quartier et pendant 3 semaines :

- Traitement 1 : Le Maïs QPM,

- Traitement 2 : Le maïs ordinaire associé au Soja,

- Traitement 3: Le maïs ordinaire,

- Traitement 4: Le témoin est représenté par les enfants non soumis au traitement.

Chaque traitement est répété trois fois en fonction des nombre des quartiers considérés. L'objectif est de voir l'influence de chaque traitement sur l'évolution des poids chez les enfants mal nutris soumis aux différents traitements et déterminer le meilleur aliment parmi ces quatre. Nourris chaque jour entre 7 heures et 9 heures du matin, il sied de signaler que ces enfants ont été pesé individuellement avant et à la fin des traitements. Ci-dessous, la préparation et le traitement à la bouillie. 


\section{RESULTATS ET DISCUSSION}

Les résultats, en termes d'évolution des poids des enfants en fonction des différents traitements sont présentés dans le Tableau 1 qui suit :

Tableau 1 : Evolution des poids des enfants en fonction de différents traitements

\begin{tabular}{|c|c|c|c|c|c|}
\hline \multirow[t]{2}{*}{ Enfant } & \multicolumn{2}{|c|}{ Avant traitement } & \multirow{2}{*}{$\begin{array}{c}\begin{array}{c}\text { Après } \\
\text { traitement }\end{array} \\
\text { Poids } \\
(\mathrm{kg})\end{array}$} & \multirow{2}{*}{$\begin{array}{c}\begin{array}{c}\text { Gain total } \\
\text { de poids }\end{array} \\
(\mathrm{kg})\end{array}$} & \multirow[t]{2}{*}{ Traitement } \\
\hline & Age (an) & $\begin{array}{l}\text { Poids } \\
\text { (kg) }\end{array}$ & & & \\
\hline \multicolumn{5}{|c|}{ Quartier Makota } & \multirow[t]{5}{*}{ Maïs QPM } \\
\hline 1 & 3 & 7,500 & 8,900 & 1,4 & \\
\hline 2 & 3 & 6,800 & 7,800 & 1 & \\
\hline 3 & 3 & 5,900 & 7,100 & 1,2 & \\
\hline \multirow[t]{2}{*}{4} & 3 & 9,200 & 10,100 & 0,9 & \\
\hline & & & & 1,125 & \\
\hline 1 & 2 & 4,300 & 4,800 & 0,5 & \multirow[t]{5}{*}{ Maïs ordinaire associé au Soja } \\
\hline 2 & 3 & 6,100 & 7,00 & 0,9 & \\
\hline 3 & 2,8 & 5,300 & 7,00 & 1,7 & \\
\hline \multirow[t]{2}{*}{4} & 2,6 & 4,100 & 4,400 & 0,3 & \\
\hline & & & & 0,85 & \\
\hline 1 & 3 & 4,400 & 4,600 & 0,2 & \multirow[t]{4}{*}{ Maïs ordinaire } \\
\hline 2 & 2,7 & 6,300 & 6,400 & 0,1 & \\
\hline 3 & 3 & 5,100 & 5,300 & 0,2 & \\
\hline \multirow[t]{2}{*}{4} & 3 & 4,100 & 4,300 & 0,2 & \\
\hline & & & & 0,175 & \\
\hline 1 & 3 & 5,00 & 5,100 & 0,1 & \multirow[t]{4}{*}{ Témoin } \\
\hline 2 & 3 & 6,00 & 5,400 & $-0,6$ & \\
\hline 3 & 2,8 & 7,00 & 7,00 & 0 & \\
\hline \multirow[t]{2}{*}{4} & 2,5 & 5,100 & 4,900 & $-0,2$ & \\
\hline & & & & -0.175 & \\
\hline \multicolumn{6}{|c|}{ Quartier Tshibangu Mpata } \\
\hline 1 & 3 & 9,00 & 10,300 & 1,3 & \multirow[t]{4}{*}{ QPM } \\
\hline 2 & 3 & 9,00 & 10,900 & 1,9 & \\
\hline 3 & 3 & 10,00 & 12,100 & 2,1 & \\
\hline \multirow[t]{2}{*}{4} & 2,8 & 4,300 & 7,00 & 2,7 & \\
\hline & & & & 2,0 & \\
\hline 1 & 2 & 4,500 & 6,00 & 1,5 & \multirow[t]{4}{*}{ Maïs ordinaire associé au Soja } \\
\hline 2 & 3 & 7,00 & 8,500 & 1,5 & \\
\hline 3 & 3 & 3,800 & 4,900 & 1,1 & \\
\hline \multirow[t]{2}{*}{4} & 3 & 8,00 & 7,00 & 1 & \\
\hline & & & & 1,27 & \\
\hline 1 & 3 & 11,00 & 11,100 & 0,1 & \multirow[t]{4}{*}{ Maïs Ordinaire } \\
\hline 2 & 3 & 7,00 & 6,500 & $-0,5$ & \\
\hline 3 & 3 & 8,00 & 8,100 & 0,1 & \\
\hline \multirow[t]{2}{*}{4} & 3 & 5,00 & 5,100 & 0,1 & \\
\hline & & & & $-0,05$ & \\
\hline 1 & 3 & 11,00 & 11,00 & 0 & \multirow[t]{3}{*}{ Témoin } \\
\hline 2 & 3 & 9,00 & 9,00 & 0 & \\
\hline 3 & 3 & 10,00 & 9,200 & $-0,8$ & \\
\hline
\end{tabular}




\begin{tabular}{|c|c|c|c|c|c|}
\hline 4 & 3 & 5,00 & 5,100 & 0,1 & \\
\hline & & & & -0.175 & \\
\hline \multicolumn{6}{|c|}{ Quartier Kamisangi } \\
\hline 1 & 3 & 9,100 & 12,00 & 2,9 & \multirow[t]{4}{*}{ QPM } \\
\hline 2 & 3 & 4,100 & 6,00 & 1,9 & \\
\hline 3 & 3 & 3,400 & 5,100 & 1,7 & \\
\hline \multirow[t]{2}{*}{4} & 3 & 8,300 & 11,00 & 2,7 & \\
\hline & & & & 2,3 & \\
\hline 1 & 3 & 8,100 & 8,900 & 0,8 & \multirow{4}{*}{ Maïs ordinaire associé au Soja } \\
\hline 2 & 3 & 6,00 & 6,900 & 0,9 & \\
\hline 3 & 3 & 6,300 & 7,00 & 0,7 & \\
\hline \multirow[t]{2}{*}{4} & 3 & 7,00 & 8,100 & 1,1 & \\
\hline & & & & 0,87 & \\
\hline 1 & 3 & 5,100 & 5,300 & 0,2 & \multirow[t]{4}{*}{ Maïs ordinaire } \\
\hline 2 & 3 & 4,300 & 4,600 & 0,3 & \\
\hline 3 & 3 & 5,00 & 5,200 & 0,2 & \\
\hline \multirow[t]{2}{*}{4} & 3 & 6,00 & 6,100 & 0,1 & \\
\hline & & & & 0,2 & \\
\hline 1 & 3 & 9,00 & 8,100 & $-0,9$ & \multirow[t]{4}{*}{ Maïs seul } \\
\hline 2 & 3 & 7,300 & 7,400 & 0,1 & \\
\hline 3 & 3 & 10,00 & 10,00 & 0 & \\
\hline \multirow[t]{2}{*}{4} & 3 & 7,00 & 6,500 & $-0,5$ & \\
\hline & & & & -0.032 & \\
\hline
\end{tabular}

Analyse Statistique: L'analyse statistique des données a été effectuée par le biais du test d'analyse de variance (ANOVA) à l'aide du logiciel Statistis 8.0.
La mise en évidence des différences significatives entre traitements a été réalisée au moyen du test LSD au seuil de $5 \%$ de probabilité.

Tableau 2 : L'analyse de variance des résultats.

\section{Traitement}

Maïs ordinaire +soja

Maïs ordinaire seul

QPM seul

Témoin

CV

Gain de poids
$09967 b$
$01083 c$
$18083 a$
$00125 c$
$4481 \%$
02654

SD

Les moyennes suivies d'une même lettre dans la même colonne ne diffèrent pas significativement au seuil de $5 \%$ de probabilité selon le test $L S D(p>0,05)$

\section{DISCUSSION}

L'analyse des moyennes en termes de gain de poids observés chez les enfants après traitement, montre que la ration constituée du traitement des enfants au maïs QPM a donné des bons résultats et a montré une différence hautement significative sur les autres traitements, Comme le confirme Mbuyi et al., 2016. La ration constituée de mais ordinaire associé au soja vient après et montre une différence significative avec les deux dernières rations. Ces résultats sont similaires à ceux réalisés par d'autres chercheurs [Subsuban et al. 1989] ; [Osei et al. 1998] ; [Onimisi et al. 2009] ; [Panda et al. 2010] ; [Tiwari et al. 2013]. Cette situation est similaire à celle de la fiche technique des performances et l'analyse de la variance a révélé une différence significative $(P<0,05)$ entre les différents aliments par rapport aux poids vifs des enfants à la fin de l'expérimentation. La différence en gain de poids entre l'aliment à base de QPM et les autres rations serait due à la valeur nutritionnelle élevée du QPM dont la digestibilité avoisine 80 à $90 \%$ celle du lait (FAO, 
1992). Le faible gain de poids observé avec le maïs ordinaire seul serait dû à une des principales limites nutritionnelles du maïs normal et son pauvre profil nutritionnel dû au déficit en acides aminés essentiels, la lysine, le tryptophane et la méthionine (Mbuya et al.,
2010). Ce travail corrobore avec celui K. MBUYA, et al. 2014 qui ont démontré que l'aliment à base de QPM peut efficacement remplacer l'aliment commercial dans la ration des poulets de chair.

\section{Le graphique suivant présente les gains de poids en fonction de chaque ration (traitement).}

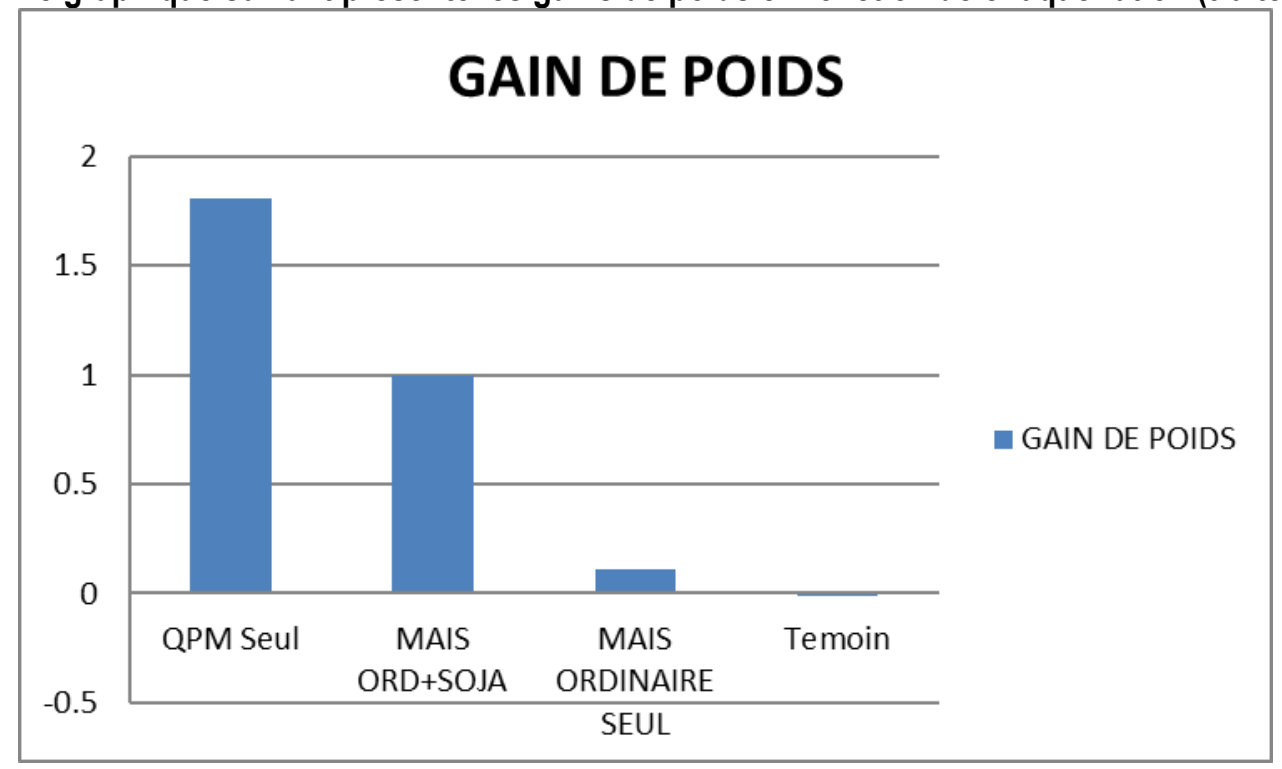

Le graphique ci-haut présenté illustre clairement les gains de poids des enfants soumis aux différents traitements ainsi que les performances de chaque ration. II ressort de ce graphique que la ration au QPM a donné le meilleur gain de poids, suivie de loin par la ration constituée de maïs ordinaire associé au soja.

\section{CONCLUSION}

Le dispositif expérimental a consisté à soumettre différents groupes d'enfants de 2 à 5 ans aux différentes rations constituées des ingrédients locaux en se basant principalement sur les rations constituées de maïs qui constitue l'aliment de base et le moins cher dans la province. Dans ce travail, l'objectif est de mener une expérience de nutrition sur les enfants de la ville de Mwene Ditu avec quatre rations constituant quatre traitements alimentaires afin de voir l'impact de la ration qui a une influence positive sur le poids des enfants. Et voir aussi quelle est la part du maïs QPM, appelé maïs viande dans cette évolution de poids des enfants. Pour ce faire, quatre rations ont été constituées et chaque ration a constitué un traitement.

$\mathrm{T} 1$ : la ration à base de maïs $\mathrm{QPM}$,

T2 : la ration à base de maïs ordinaire associé au soja,

$\mathrm{T} 3$ : la ration à base de maïs ordinaire seul, T4 : le témoin (sans traitement).
Tandis que la ration au maïs ordinaire ainsi que le témoin n'ont pas significativement influencé les gains de poids des enfants. De ce qui précède, l'étude recommande la multiplication de QPM afin que ce maïs soit utilisé dans la récupération des enfants malnutris.

Chaque ration était distribuée à un groupe de quatre enfants dans les trois quartiers choisis de la ville de Mwene Ditu. Les enfants étaient nourris à la bouillie chaque matin durant trois semaines et chaque enfant avaient été pesé au début de l'expérience ainsi qu'à la fin de la période de nutrition qui a duré trois semaines. II ressort, après l'analyse des moyennes que la ration à base de mais QPM a entrainées une forte augmentation des poids des enfants dans tous les groupes et a montré une différence hautement significative par rapport à toutes les autres rations, suivie de la ration à base de maïs ordinaire mélangé au soja. La ration à base du maïs ordinaire ainsi que le témoin n'ont rien contribué dans l'augmentation des poids des enfants ayant été soumis à ces traitements. Là où on ne dispose pas le QPM, on peut utiliser le maïs ordinaire mélangé au soja malgré le prix élevé du soja sur le marché par rapport au maïs. 


\section{REFERENCES BIBLIOGRAPHIQUES.}

Aubry P., 2009. Malnutrition protéino-énergétique et avitaminose.

Beaufrere B., Bresson JL., Briend A., 1998. Besoins en protéines et en énergie de l'enfant atteint de malnutrition sévère. Applications au traitement en milieu hospitalier de la malnutrition par carence d'apport.

Belchior-Beliuno V, 2002. La prise en charge pratique des problèmes nutritionnels et alimentaires en situation de crise. Méd. Trop.

Briend A. 2003. Prise en charge de la malnutrition sévère de l'enfant en milieu tropical. Méd. Trop.

FAO, 1970. Tables des compositions des aliments à l'usage de l'Afrique

FAO, 2017. L'avenir de l'alimentation et de l'agriculture. Tendance et défit, Rome.

Jeliff D.B., 1957. L'alimentation des nourrissons dans les régions tropicales.

Jeliff D.B., 1957. La nutrition de l'enfant en voie d'équipement-collection techniques américaine № 136 USAID.

Mbuya K., 2015. Présentation sur le QPM lors du lancement National des variétés QPM en $\mathrm{RDC}$.

Mbuya K, Nyebo K. Bombani B. 2015. Potentialité des rations formulées à base des ingrédients locaux pour la volaille dans la Province du Kasaï Oriental.1626-1631.

Subsuban CP, Campbell IH, and Pelagio O. [1989]. Comparative evaluation of quality protein maize (QPM) and normal maize in animal feed. In: FAO Animal Production and Health Proceedings: Protein Sources for Animal Feed Industry. Expert Consultation and Work shop. Bangkok, 29 April to 3 May.

Onimisi P.A., Omage J.J., Dafwang I.I. and Bawa G.S. [2009]. Replacement Value of Normal Maize with Quality Protein Maize (Obatampa) in
Broiler Diets. Asian Network for Scientific Information, Pakistan Journal of Nutrition 8 (2): 112-115.

Osei S.A., Atuahene C.C, Okai D.B., Donkoh A. And Tuah A.K. [1998]. The nutritive value of quality protein maize in the diets of broilers chickens. Ghan Jnl agr.Sci 31:1-5. Accra National Science \& Technology Press.

Panda A.K., Raju M.V.L.N., Rama Rao S.V., Lavanyag.

Reddy P.K.E and Shyam Sunder G. [2010]. Replacement of normal maize with quality protein maize on performance, immune response and carcass characteristics of broiler chickens. Asian-Aus J Anim Sci 23 (12): 1626-1631.

Prudhon C., 2001. La malnutrition en situation de crise. Manuel de prise en charge thérapeutique et de planification d'un programme nutritionnel. Editions Karthala et Action contre la faim, 342.

Raoul A., 1970. Les centres de récupération nutritionnels.

Rotsart I. Et Courtejoie J., 1992. Malnutrition, faim des protéines.

Rotsart I. Et Courtejoie J., 1992. Nutrition, l'éducation nutritionnelle dans la pratique journalière.

Senecal J., Dupin A., 1971. Les besoins nutritionnels de l'enfant au cour des premières années.

Senecal J., 1972. La malnutrition protéino-calorique ou marasme-Kwashiorkor chez les enfants en milieux Tropical.

Tiwari M.R., Chapagain P.B., Shah M.K. And Shrestha Y.K. [2013]. Evaluation of Quality Protein Maize (QPM) and Normal Mazie for Growth Performance of Crossbred piglets in Wester Hills of Nepal. Global Journal of Science frontier research Agriculture and Veterinary 13(6):1-7.

Williams C., 1935. Le Kwashiorkor du sevrage. 